in both 7-week-old and 18-week-old mice that were already sick, although an improvement in survival was only observed in the older group.

This therapy has obvious clinical applications, considering that the appearance of antiDNA autoantibodies often precedes the onset of human disease by many years. The efficacy in older mice demonstrates that this therapy could also be used to treat patients already displaying SLE symptoms.

Original article Tchorbanov AI et al. (2007) Selective silencing of DNA-specific B lymphocytes delays lupus activity in MRL/lpr mice. Eur J Immunol 37: 3587-3596

\section{Oligonucleotide IRS 954 might represent a novel therapy for SLE}

In the autoimmune disease systemic lupus erythematosus (SLE), stimulation of Toll-like receptors (TLR) 7 and 9 by self nucleic acids is postulated to induce plasmacytoid dendritic cells (PDCs) to overproduce interferon- $\alpha$, a key cytokine in the pathogenesis of SLE. TLR7 and TLR9 are also thought to be involved in B-cell-mediated production of autoantibodies specific to nucleic acids, thereby triggering and then promoting SLE.

Barrat et al. have described previously an oligonucleotide-based inhibitor of TLR7 and TLR9, called immunoregulatory sequence (IRS) 954, which can block B-cell activation and interferon- $\alpha$ production by PDCs. Using the $(\mathrm{NZB} \times \mathrm{NZW}) \mathrm{F} 1$ mouse model of SLE, they have now investigated its potential as a therapy for SLE. Mice were injected twice weekly with IRS 954 from the age of 4 months, when they normally start showing signs of SLE. At the age of 9 months, IRS 954-treated mice had significantly lower serum levels of nucleicacid-specific autoantibodies than untreated mice. They also exhibited decreased proteinuria, reduced glomerulonephritis and end-organ damage, and increased survival.

The authors conclude that IRS 954 is able to reduce symptoms and prolong survival in the $(\mathrm{NZB} \times \mathrm{NZW}) \mathrm{F} 1$ mouse model of SLE, supporting the hypothesis that blockade of TLR7 and TLR9 in both B cells and PDCs could be used as a novel therapeutic strategy for the treatment of SLE.

Original article Barrat FJ et al. (2007) Treatment of lupusprone mice with a dual inhibitor of TLR7 and TLR9 leads to reduction of autoantibody production and amelioration of disease symptoms. Eur J Immunol 37: 3582-3586

\section{A simple scoring algorithm to identify men at risk for osteoporosis and hip fracture}

The risk of hip fracture for women with osteoporosis can be reduced by early identification and treatment. Guidelines for primary screening of men, however, are lacking and, although hip fracture is less common in men, it is associated with higher mortality. Shepherd et al., therefore, developed and validated a clinical prediction rule to identify US men at increased risk of osteoporosis and hip fracture.

Data of 2,995 men (aged $\geq 50$ years) from the National Health and Nutrition Examination Survey III who had valid dual-energy X-ray absorptiometry (DXA) data were randomly allocated to a development $(n=1,497)$ or validation $(n=1,498)$ cohort. A best-fitting multivariable logistic regression analysis identified three important predictive variables for osteoporosis-age, weight and history of chronic obstructive pulmonary disease, with low weight $(\leq 70 \mathrm{~kg})$ having the strongest predictive validity. These three variables were included in a simple scoring algorithm to determine which men should be referred for DXA. A Male Osteoporosis Risk Estimation Score (MORES) of $\geq 6$ correctly identified $93 \%$ of the men with osteoporosis in the overall cohort and had high predictive ability in the validation cohort. Furthermore, a simulated number-needed-to-screen analysis was favorable, especially for older age-groups ( $\geq 60$ years).

The authors conclude that the MORES performs well compared with other available risk assessment guidelines for men, and is a valid approach for men aged $\geq 60$ years that has the potential to reduce osteoporotic fractures and associated mortality.

Original article Shepherd AJ et al. (2007) Development and internal validation of the male osteoporosis risk estimation score. Ann Fam Med 5: 540-546 